\title{
Disinhibition Outside Receptive Fields in the Visual Cortex
}

\author{
Gary A. Walker, Izumi Ohzawa, and Ralph D. Freeman \\ Group in Vision Science, School of Optometry, University of California, Berkeley, Berkeley, CA 94720-2020
}

By definition, the region outside the classical receptive field (CRF) of a neuron in the visual cortex does not directly activate the cell. However, the response of a neuron can be influenced by stimulation of the surrounding area. In previous work, we showed that this influence is mainly suppressive and that it is generally limited to a local region outside the CRF. In the experiments reported here, we investigate the mechanisms of the suppressive effect. Our approach is to find the position of a grating patch that is most effective in suppressing the response of a cell. We then use a masking stimulus at different contrasts over the grating patch in an attempt to disinhibit the response. We find that suppressive effects may be partially or completely reversed by use of the masking stimulus. This disinhibition suggests that effects from outside the CRF may be local. Although they do not necessarily underlie the perceptual analysis of a figure-ground visual scene, they may provide a substrate for this process.

Key words: visual cortex; receptive field; nonclassical receptive field; figure-ground; disinhibition; single cortical neurons
Behavioral tests of human subjects demonstrate that the spatial and temporal context of a visual stimulus may exert a clear influence on perception. For example, flanking contours can reduce stereoscopic acuity, and effects can be observed for both spatial and temporal parameters (Butler and Westheimer, 1978). Physiological studies also suggest the role of context in the responses of single neurons. Although the classical receptive field (CRF) is, by definition, the only area within which one can activate an individual fiber or neuron, the region beyond this area can modulate the response (for review, see Allman et al., 1985). Some cells have been reported to respond more vigorously when the stimulus patterns within and outside the CRF are perceptually different. These response modulations have been observed in neuronal activity associated with perceptual "pop-out" or "figureground" stimuli (Nothdurft, 1991; Knierim and van Essen, 1992; Lamme, 1995; Zipser et al., 1996; Kastner et al., 1997). In these cases, neuronal discharge is weaker when the CRF and surround areas (center and background) are perceptually identical. Reports have also been made of increased neuronal responses when perceptually relevant line segments are presented outside the CRF (Polat et al., 1998). Furthermore, reversible inactivation of MT suggests a possible feedback role in figure-ground segregation (Hupe et al., 1998).

The notion of contextual modulation of visual processing, based on figure-ground image analysis, is appealing. However, it is possible that simple local mechanisms, such as those involved in cross-orientation suppression (DeAngelis et al., 1992), may account for the reported findings. In a local mechanism, an inhibitory effect is solely dependent on stimuli in local regions gener-

\footnotetext{
Received Oct. 30, 2001; revised March 21, 2002; accepted March 25, 2002.

This work was supported by National Eye Institute Research and CORE Grants EY01175 and EY3176.

Correspondence should be addressed to Ralph D. Freeman, 360 Minor Hall, University of California, Berkeley, Berkeley, CA 94720-2020. E-mail: freeman@ neurovision.berkeley.edu.

G. A. Walker's present address: Guidant Corporation, 1525 O’Brien Drive, Menlo Park, CA 94025.

I. Ohzawa's present address: Department of Biophysical Engineering, Osaka University, 1-3 Machikaneyama, Toyonaka-shi, Osaka 560-8531, Japan.

Copyright (C) 2002 Society for Neuroscience $0270-6474 / 02 / 225659-10 \$ 15.00 / 0$
}

ating the inhibitory signal. The strength of surround inhibition is strictly related to activation levels of cells with CRFs in the surround area. Regarding contextual effects in primary visual cortex, results of a recent study suggest that V1 neurons do not segregate figures from ground (Rossi et al., 2001).

Aside from perceptual implications, the nature of interactions between the CRF and surround areas is of primary interest. We conducted the experiments described here to investigate the characteristics of the local inhibitory surround effects that we identified previously (DeAngelis et al., 1994; Walker et al., 1999, 2000). Specifically, can the suppressive effects of regions outside the CRF be reversed by use of appropriate stimuli? We are also interested in potential mechanisms both within and outside the CRF that may account for interactive effects.

Our primary finding is as follows. When a cell is suppressed by stimulation outside the CRF, a reversal of this effect may be brought about by the addition of a masking stimulus positioned in the surrounding region of the CRF. This disinhibition can restore the response of the cell to the level obtained with optimal stimulation of the CRF by itself. We propose that the suppression from the surrounding region of the CRF is distinct from the contrast normalization process that is thought to be a primary mechanism within the CRF (Heeger, 1992; Carandini et al., 1997, 1999).

\section{MATERIALS AND METHODS}

Physiological preparation. Experiments were conducted using anesthetized, paralyzed cats. Before anesthesia, acepromazine maleate $(0.5$ $\mathrm{mg} / \mathrm{kg})$ and atropine sulfate $(0.06 \mathrm{mg} / \mathrm{kg})$ are injected subcutaneously to provide tranquilization and to suppress secretion, respectively. Anesthesia is induced and maintained during surgery with $2-4 \%$ isoflurane. Forepaw femoral veins are cannulated for intravenous infusion, a tracheal tube and a rectal thermometer are inserted, and electrocardiographic (ECG) leads and electroencephalographic (EEG) screw electrodes are placed. A craniotomy is performed around Horsley-Clarke coordinates P4L2, and the dura is carefully removed. Commercial insulated tungsten microelectrodes are positioned just above the surface of the cortex. The craniotomy is filled with agar and sealed with wax.

Animals are artificially respirated at $\sim 25$ strokes/min with a mixture of $\mathrm{N}_{2} \mathrm{O}(70 \%)$ and $\mathrm{O}_{2}(30 \%)$. Anesthesia and paralysis are maintained by intravenous infusion of a mixture of thiopental sodium (Pentothal, $2.5 \%$ 
solution; $1.4 \mathrm{mg} \cdot \mathrm{kg}^{-1} \cdot \mathrm{hr}^{-1}$ ) and gallamine triethiodide (Flaxedil, $2 \%$ solution; $\left.9.4 \mathrm{mg} \cdot \mathrm{kg}^{-1} \cdot \mathrm{hr}^{-1}\right)$, combined with a $5 \%$ dextrose and lactated Ringer's solution $\left(0.5 \mathrm{ml} \cdot \mathrm{kg}^{-1} \cdot \mathrm{hr}^{-1}\right)$. Steady-state hydration is provided by a drip system through which lactated Ringer's solution is infused $\left(10 \mathrm{ml} \cdot \mathrm{kg}^{-1} \cdot \mathrm{hr}^{-1}\right)$. Core body temperature is maintained near $38^{\circ} \mathrm{C}$, and end-tidal $\mathrm{CO}_{2}$ at $4-4.5 \%$. EEG, ECG, heart rate, core body temperature, and expired $\mathrm{CO}_{2}$ are monitored continuously. The pupils are dilated with $1 \%$ atropine sulfate, and nictitating membranes are retracted with $5 \%$ phenylephrine hydrochloride. Contact lenses (+2D) with $4 \mathrm{~mm}$ artificial pupils are placed on both corneas. The contact lenses are removed and cleaned periodically, and the clarity of the refractive media is checked with a direct ophthalmoscope. Chloromycetin (1.50 $\mathrm{ml} / \mathrm{d}$ ) is given prophylactically every $12 \mathrm{hr}$.

Experimental apparatus. Visual stimuli are displayed on a tangent screen in front of the animal or on two separate cathode ray tube (CRT) displays, allowing independent stimulation of each eye. A manually controlled joystick is used in preliminary tests of the receptive field (RF) to sweep a bar stimulus of variable size and orientation in any position and direction.

A visual stimulator generates images on each CRT display independently. The stimulator consists of a personal computer with two highresolution graphics boards and runs custom software. The frame refresh rate of each CRT display is $76 \mathrm{~Hz}$, and both displays are refreshed synchronously. Stimuli are delivered with a temporal resolution of one frame period $(13.2 \mathrm{msec})$ by custom temporal modulation driver software. The spatial resolution is $1024 \times 804$ pixels. The usable portion of the display subtends an area of $28 \times 22^{\circ}$ (viewed at $57 \mathrm{~cm}$ ), and the mean luminance at the front surface of each contact lens is $23 \mathrm{~cd} / \mathrm{m}^{2}$.

For presentations requiring two superimposed gratings, the component gratings are displayed on alternate scan lines (line interleaving) to avoid any interaction of the two components resulting from the bandwidth limitations of the video amplifiers in the displays (Pelli and Zhang, 1991). This line interleaving method of producing a plaid results in an effective contrast that is $50 \%$ of the nonline interleaving contrast. Thus, an $80 \%$ contrast grating without line interleaving is a $40 \%$ contrast grating when presented with line interleaving.

Microelectrodes are inserted via a guide tube and advanced through the cortex by a piezoelectric micropositioner. Custom-made digital signal-processing software is used to discriminate individual action potentials. This software allows accurate discrimination of individual spikes from multiple cells. After discrimination, each action potential is recorded as a binary event, time stamped with $1 \mathrm{msec}$ accuracy, and stored for analysis off-line.

Procedures. A cell is encountered, and the spike waveform is isolated. Location and approximate orientation preference of the CRF are determined. An interactive search program (DeAngelis et al., 1993) is then used to determine suitable parameters for a circular patch of drifting sinusoidal grating. The grating patch is presented on the CRT, and the size, orientation, and spatial frequency of the grating are adjusted by the experimenter to determine preferred values.

The subjective tests are followed by quantitative analysis of the CRF. Grating stimuli are presented monocularly for $4 \mathrm{sec}$ at a time (temporal frequency is $2 \mathrm{~Hz}$ for all gratings) in blocks of randomly interleaved trials. The size of the stimulus for these initial presentations is typically $5-8^{\circ}$ in diameter Each stimulus is presented at least four times, and successive presentations are separated by $3 \mathrm{sec}$, during which the animal views blank screens of the same mean luminance as the gratings. After presentation of a complete set of stimuli, the DC (mean rate) and first harmonic (at $2 \mathrm{~Hz}$ ) components of the accumulated response are computed for each stimulus. Response amplitude is defined as the greater of the mean firing rate or the amplitude of the first harmonic of the response. Simple and complex cell designations are determined by classical criteria (Hubel and Wiesel, 1962) and by the ratio of the first harmonic and mean of the response to a drifting grating stimulus (Skottun et al., 1991).

Orientation tuning of the CRF is determined by use of a series of drifting grating stimuli, differing in orientation around the initial orientation estimate. Spatial frequency and size for this run are set to the initial values obtained using the search program. The peak of the resultant tuning curve is used as the optimal orientation for subsequent presentations. Preferred spatial frequency for the cell is similarly determined.

Optimal orientation, spatial frequency, and size for CRF stimulation were determined quantitatively for each cell from the preliminary runs. In this work, "optimal stimulus" is used to refer to a drifting grating with orientation, spatial frequency, and size parameters set to the values that elicit the greatest response from the cell. Contrast was set at an intermediate value that varied from cell to cell but was typically $\sim 30 \%$. Sinusoidal gratings were drifted for $4 \mathrm{sec}$ at a temporal frequency of 2 $\mathrm{Hz}$. After the optimal stimulus was determined for each cell, the size of the CRF was estimated by presenting a drifting grating within a circularly bounded window of variable size. The resultant size-tuning curve yields an estimate of the spatial dimensions of the CRF and also the degree of surround suppression (Walker et al., 2000).

Spatiotemporal maps of the CRF were also obtained for some cells using either the reverse correlation (DeAngelis et al., 1993) or m-sequence (Sutter and Tran, 1992; Anzai et al., 1997) methods. These maps were used to verify the accuracy and reliability of the parameters obtained with grating stimuli. One particular advantage of these maps is that they provide very accurate information about the center and size of the CRF. In general, we find excellent agreement between the grating and noise measurements.

\section{RESULTS}

Results reported here come from a pool of multiprotocol experiments. For the current study, we completed 29 monoptic protocol runs from 24 cells (nine simple and 15 complex). From five cells, tests were run for left and for right eyes. These 24 cells were studied because they all exhibited some degree of surround suppression.

Experimental predictions based on alternative notions of $\mathrm{RF}$ surround organization are illustrated in Figure 1. In Figure $1 A_{1}$, the CRF is stimulated with an optimal grating, causing a strong response from the cell. Predicted responses (action potentials) are depicted schematically by vertical lines under each RF icon. In $A_{2}$, the cell is also stimulated by an optimal grating, but in this case, it covers the CRF plus the surround. The surround is inhibitory, and the response of the cell is therefore reduced compared with that in $A_{1}$. In $A_{3}$ and $A_{4}$, gratings are presented outside the CRF at optimal and orthogonal orientations, respectively. The cell does not respond in either case. In $A_{5}$, the region outside the CRF is covered by two gratings, one at an optimal orientation and a second at an orthogonal one. As before, these outer gratings do not drive the cell because they are not within the CRF. Because surround suppression is generally tuned to an orientation that matches the preferred value of the CRF (DeAngelis et al., 1992; $\mathrm{Li}$ and $\mathrm{Li}, 1994)$, the orthogonal grating $\left(A_{4}\right)$ is not likely to cause suppression. However, perceptually, the pair of orthogonal gratings constitutes a plaid pattern that is distinct from that of the grating within the CRF. It therefore constitutes a central figure (the optimal grating) against a plaid texture background. The figure-ground notion is that RF surrounds are required to differentiate between salient features of a scene. The figure of attention is presumed to activate cortical neurons selectively, whereas background components cause weak activity. Therefore, according to the figure-ground model of $A_{5}$, the cell exhibits a strong response. The alternative model, which we designate "disinhibition," also indicates a vigorous response. In this case, cells in the region outside the CRF are stimulated by the large grating of optimal orientation, and the activity in these "surround" cells is relayed to the "center" cell via inhibitory connections. However, the suppression is neutralized by the orthogonal grating attributable to cross-orientation suppression (Morrone et al., 1982; Bonds, 1989; DeAngelis et al., 1992; Walker et al., 1998). Cells contributing to surround suppression are therefore inactivated or disinhibited to some degree by the presence of the additional orthogonal grating. Therefore, the reduced suppression results in a strong response from the cell whose CRF is within the surround.

The conditions illustrated in Figure $1 B$ are similar to those of 
A

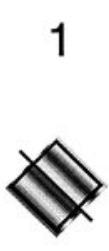

2
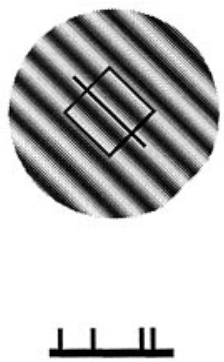

B 1

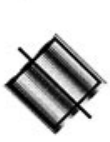

IIIIIIIIIL

C

1
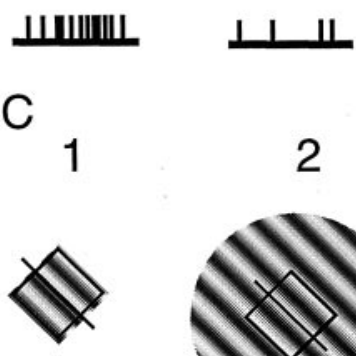

2
3
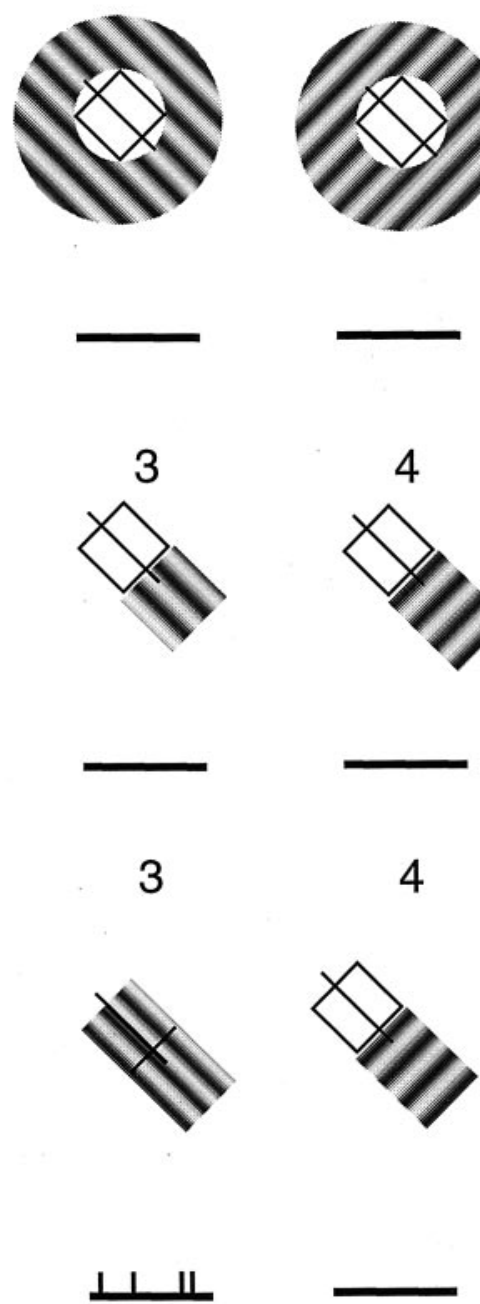

4

$\perp \perp$

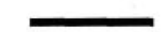

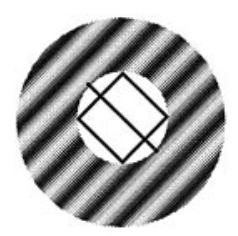
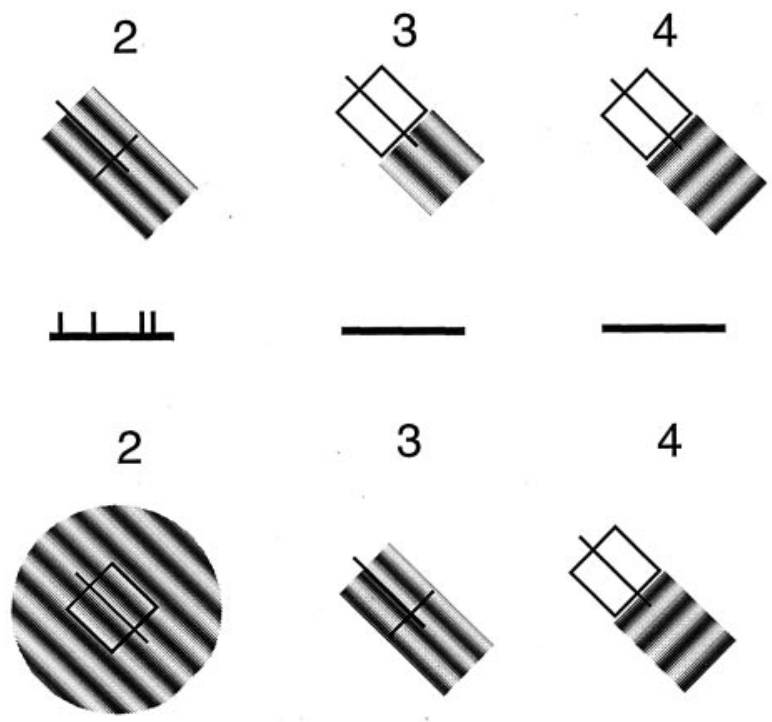

Lلـ

LIIIIIIII

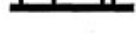

\section{ \\ ㅍIIIㄴ Disinhibition}

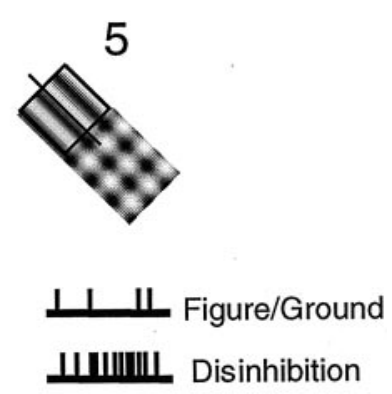

5

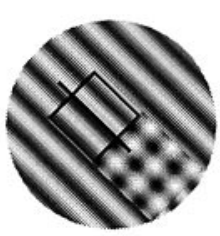

\section{Lلـ Figure/Ground لIIIIIIIL Disinhibition}

Figure 1. RF configurations are shown for different conditions of stimulation of the CRF and the surround. CRFs are represented as squares with lines through them to indicate preferred orientations. Predicted responses for given conditions are depicted below each RF as schematic spike discharges. In $A_{1}$, the cell is activated by a drifting grating of optimal parameters that fills the CRF. In $A_{2}$, a large grating of the same parameters covering the CRF and surround elicits a relatively weak response attributable to suppression from the area outside the CRF. Stimulation of the surround alone, in $A_{3}$ (at optimal orientation) and $A_{4}$ (at an orthogonal orientation), produces no response. Combining both surround orientations (stimuli $A_{2}, A_{4}$ ), producing the plaid surround and optimal orientation center in $A_{5}$, causes a response equivalent to that in $A_{1}$. This result is predicted for both figure-ground and disinhibition models. In $B_{1}$, optimal stimulation of the CRF is shown again. Suppression of the optimal response is indicated in $B_{2}$ by the addition of a section of optimal grating into a suppressive surround region on one end of the CRF. The same section of grating, at optimal and orthogonal orientations $\left(B_{3}, B_{4}\right)$ presented outside the CRF causes no response. The combination of patterns $B_{2}$ and $B_{4}$, shown in $B_{5}$, causes different response strengths for figure-ground and disinhibition models. In $C_{1}$, optimal stimulation of the CRF is shown again as is the suppression of the optimal response by the large grating in $C_{2}$. In $C_{3}$, this cell is equally suppressed by a grating that is limited to one "end" of the surround. Stimulation of the end region alone with a grating orthogonal to the optimal yields no response $\left(C_{4}\right)$. Finally, stimulation of the center and surround with a large grating of optimal parameters, together with an orthogonal grating over the suppressive end causes different responses for figure-ground and disinhibition models.

Figure $1 A$, except that the stimuli outside the CRF are limited to a small region from which suppression of the cell has been localized instead of being presented in the entire surround. This configuration is of interest because many cells receive surround inhibition from a small localized area neighboring the CRF (Walker et al., 1999). In $B_{1}$, the CRF is activated by an optimal grating. The grating is extended in $B_{2}$ but only from one edge of the CRF. This suppresses the response of the cell as in the case of the full surround shown in Fig. $A_{2}$. Grating patches outside the CRF, depicted in $B_{3}$ and $B_{4}$, do not activate the cell. In $B_{5}$, a combination of stimuli $B_{2}$ and $B_{4}$, is depicted. The predicted response of the cell to an optimal grating in the CRF together with the surround patch is different for the figure-ground and disinhibition models. Because there is no salient figure-ground distinction, the response of the cell should be relatively weak. However, the disinhibition model predicts a strong response because the orthogonal grating patches outside the CRF should neutralize each other.

In Figure $1 C_{1}$, an optimal grating fills the CRF and elicits a strong response. A large optimal grating $\left(C_{2}\right)$ covering the $\mathrm{CRF}$ 
and surround yields a relatively weaker response. If the cell has an asymmetrical surround as described previously (Walker et al., 1999), then a small portion of the surround can suppress the response to the same degree as that of the entire annulus $\left(C_{3}\right)$. A grating that is orthogonal to the optimal, positioned outside the $\mathrm{CRF}$, has no effect $\left(C_{4}\right)$, and the cell does not respond. In $C_{5}$, a combination of stimuli $\left(C_{2}\right.$ and $\left.C_{4}\right)$, a large optimal grating covers the CRF and surround. In addition, a small orthogonal grating patch is positioned on one side of the surround. Assuming that stimulation of this region with an optimal grating causes suppression of the response, then the addition of an orthogonal (crossorientation) patch should reverse the suppression. This effect is predicted by the disinhibition model, and, in this case, the cell should exhibit a strong response. However, in the figure-ground model, a poor response is predicted because only a small local patch of the surround is clearly different from the CRF and the "figure" (plaid) is outside the CRF in this case (Lamme, 1995).

\section{Full-field stimulation and masking}

Figure 2 shows responses from a complex cell $(A)$ and two simple cells $(B, C)$. Testing conditions include stimulation of the CRF alone (icon 1), the CRF plus surround (icon 2), and the CRF plus surround, which contains an orthogonal grating in addition to the optimal one to form a plaid (icon 3). The experiments were performed at seven or eight contrast levels for the orthogonal surround grating. With increased contrast of the orthogonal grating, the perceptual saliency of the plaid becomes stronger so that, when the contrasts are matched, the image attains a strong figureground appearance, as illustrated in icon 3. At the highest contrasts, the orthogonal grating dominates the surround, although the plaid is still apparent. Contrasts used for the optimal grating were $30 \%$ for the two cells shown in $A$ and $B$ and $20 \%$ for the cell given in $C$.

In general, the data show two trends. First, when the orthogonal surround grating is absent, or of low contrast, the cell responds well below the level obtained with center stimulation alone. This is an example of standard surround suppression. Second, when the contrast of the orthogonal grating equals or exceeds that of the optimal grating (Fig. 2, upward arrow on $x$-axis), the response increases, i.e., is disinhibited.

To explore a range of disinhibition effects, the tests were conducted using contrast levels for the CRF grating that elicited robust responses but which did not saturate. The resulting discharge rates were maintained during the multiple measurements. Generally, we used contrast levels of $\sim 30 \%$. We refer to the response levels elicited in this manner as "optimal." In fact, greater responses could be obtained in some cases using higher contrast gratings. Examples of the range of disinhibition that we observed are shown in Figure 2. For the complex cell shown in $A$, the response is fully disinhibited by the orthogonal grating at the highest contrasts. In other words, the response is equal to that for stimulation of the CRF alone. The net effect is slightly more than a doubling of the response compared with the full-field optimal grating. The simple cell shown in $B$ provides an example of partial disinhibition. This cell is completely suppressed by the optimal surround stimulus. The disinhibition is robust, but the cell only recovers approximately half of the optimal response. The simple cell shown in $C$ was tested through both the dominant and nondominant eyes. Strong suppression is observed through the dominant left eye, and minor disinhibition is seen. For the nondominant right eye, the disinhibition is approximately equivalent in terms of the change in spikes per second, but, because the response rate is much lower, the disinhibition provides a complete recovery of the optimal response for stimulation through that eye.

To summarize the results across the population of cells studied, each response is normalized to the response of the full-field optimal grating. By using this stimulus as the baseline, i.e., the cell is in a suppressed state, we determine the effect of the orthogonal grating. Figure 3 shows the population results for 29 data sets. In Figure $3 A$, the amount of change in response is given for each cell for different contrast levels of the orthogonal surround grating. These plots illustrate two general properties that are observed for nearly every cell. At low contrasts, the orthogonal grating has no effect, and responses remain suppressed. At moderate and high contrasts, the orthogonal grating has a clear disinhibition effect. For example, at $32 \%$ contrast, when the orthogonal and optimal gratings are of nearly equal strength in the surround, 23 of 29 cells show an increase in response relative to the suppressed state. At the highest contrast level, 64\%, 26 of 29 cells respond above the suppressed baseline level. Table 1 shows all of the response increases and decreases at different contrast levels.

In Figure $3 A$, the median values of the population are indicated at each contrast level by the triangles (slightly shifted to the right for clarity). The median is plotted because it is a more conservative estimate of the effect than the mean. The mean indicates a larger effect, but it is heavily weighted by the cells with pronounced disinhibition. To compare the population distributions at various contrasts, the data at $1 \%$ contrast are used as the null conditions, and nonparametric sign-rank tests have been performed for all pairwise combinations. The distributions are not significantly different at the three lowest contrasts $(p \gg 0.05$ for 2,4 , and $8 \%$ ) but are different for the highest contrasts at high significance levels $(p<0.02$ at $16 \% ; p<0.02$ at $32 \%$; $p<0.008$ at $64 \% ; p<0.002$ at $80 \%)$. These data demonstrate that the disinhibition effect can be very substantial. For our population, the addition of high contrast masking gratings results in a nearly doubling of the response of the median cell (1.89).

In Figure $3 B$, a histogram is given showing the number of cells with increased (open bars) or decreased (shaded bars) responses when an orthogonal grating of different contrasts is superimposed on the optimal grating in the surround region of the CRF. To convey an indication of the extent of these effects, we consider an arbitrary criterion as follows. Disinhibition that causes a doubling or greater of response strength is indicated in Table 1. In Figure $3 C$, the strength of suppression is plotted against the strength of disinhibition. A suppression index is used as a metric for suppression strength, and a disinhibition index is computed as a measure of disinhibition strength. The scatter plot is fit using linear regression and indicates that, to a first approximation, the effect of disinhibition is equivalent across all cells, regardless of the strength of surround suppression.

The robust effects described above are consistent with both figure-ground and disinhibition models because both predict stronger responses in the full surround configuration (Fig. 1 $A_{5}$ ). These results demonstrate that a nonpreferred stimulus that does not drive the cell, when placed in a portion of the visual field that the cell does not respond to, causes the response of the cell to increase. In the figure-ground model, high-level feedback mechanisms are proposed to account for this effect (Zipser et al., 1996; Hupe et al., 1998). To further explore CRF and surround interactions and to differentiate between figure-ground and disinhibition models, we conducted tests in which only selected portions of the surround are stimulated. 


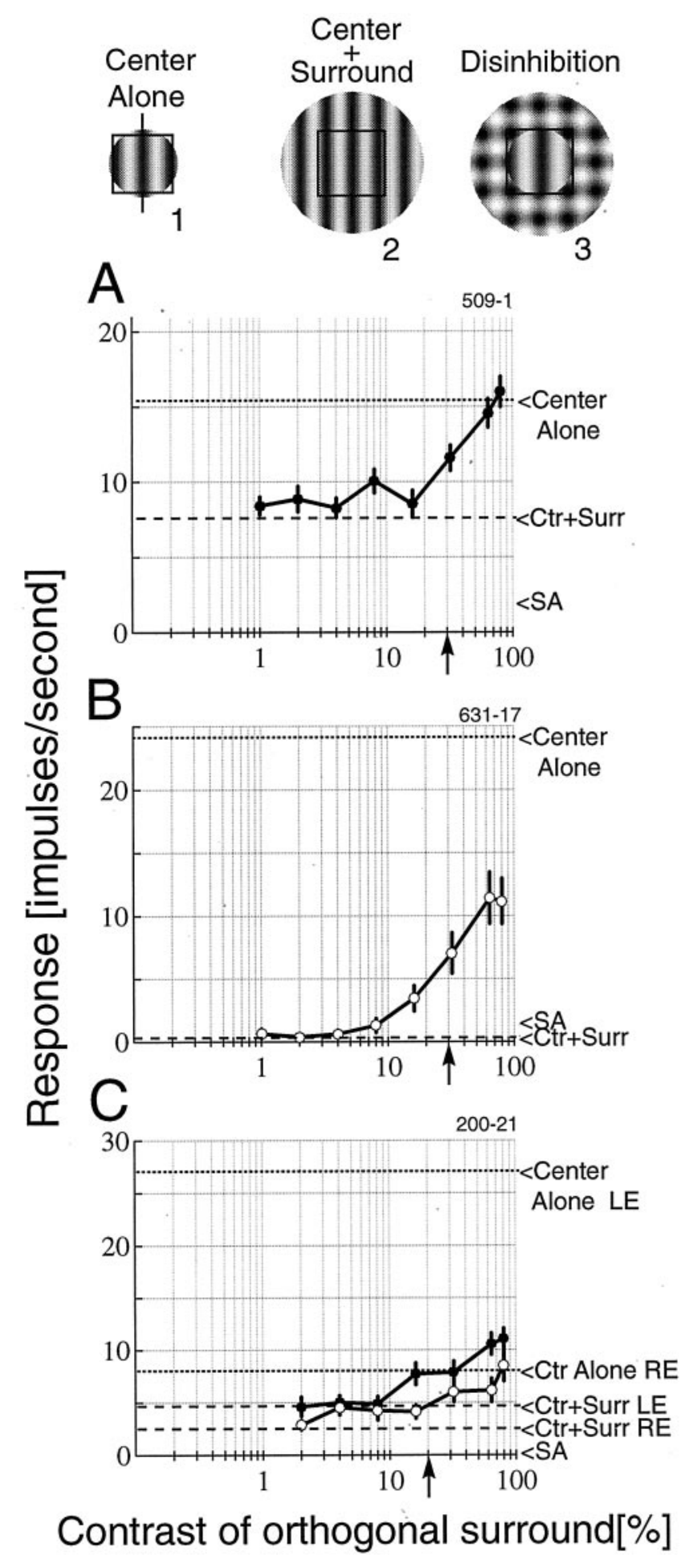

Figure 2. Three examples are illustrated of the effect of contrast of the surround grating on the responses of cells. Examples of typical stimuli are shown above the plots and represent the Center Alone (icon 1) and Center + Surround $($ Ctr + Surr; icon 2) baseline measure shown on the plots by dashed lines. Also shown is an example of the disinhibition stimulus (icon $3)$. This example depicts the test pattern when the orthogonal grating matches the contrast of the optimal grating. $A$, A complex cell exhibits strong suppression that is fully disinhibited with a high-contrast orthogonal grating. $B$, A simple cell is completely suppressed by the optimal surround. The response is strongly disinhibited but only recovers approximately half of the optimal response. $C$, Responses of a binocular simple cell are illustrated in which both eyes exhibit surround suppression and

\section{Partial surround masking}

In previous work, we determined that, for most cortical cells, a relatively small region outside the CRF is as effective in suppressing the response of a cell as an entire annulus (Walker et al., 1999). We therefore decided to see whether the specific small region outside the CRF can be disinhibited, as in the case of the full annulus. Figure 4 illustrates disinhibition for a cell under two different surround configurations. In the first case $(A)$, the cell was stimulated with a full-field $\left(15^{\circ}\right.$ diameter $)$ optimal grating covering the center and surround. As the graph shows, complete disinhibition of the response of the cell was obtained with this configuration. In the second case $(B)$, the experiment was repeated, but with the orthogonal grating presented only to the suppressive portion of the surround. This suppressive portion was determined from previous surround mapping runs (Walker et al., 1999). All of the surround suppression for this cell arises from the bottom portion of the surround (see icon 3). Therefore, a second stimulus configuration was used for the disinhibition test, as shown in icon 4. The optimal grating covers the CRF and extends into the suppressive end. The orthogonal grating covers only the suppressive region. Again, complete disinhibition is found. This indicates clearly that it is not necessary to stimulate the entire surround to induce both surround suppression and disinhibition. It also shows that figure-ground saliency is not required to obtain the same effect as that for Figure $4 A$ (for which figure-ground saliency is high). Note that, for both stimulus configurations, the response reached the level obtained with the optimal grating presented to the CRF alone.

To ensure that our surround stimulus was not directly driving the cell, we included the following control condition. On separate trials, an orthogonal grating was presented in the surround at the same contrasts as for the test conditions, with or without the optimal grating restricted to the CRF. This allowed us to examine the effect of the orthogonal grating by itself. In most cases, orthogonal gratings had no effect on the cells; they neither increased nor decreased the response at any contrast. In a few cases, the orthogonal grating caused suppression. Generally, this was attributable to an error in centering, and the suppression disappeared when the experiment was repeated with new center coordinates. However, in two cases, cells were suppressed by orthogonal surround stimuli and exhibited disinhibition when the orthogonal grating was combined with the optimal grating in the surround. For the stimulus configurations shown in Figure 4, the control condition had negligible effect on the response of the cell. The mean of the response to the control condition at all contrast levels is indicated by solid horizontal lines on the plots.

Figure 5 summarizes the results obtained when only a portion of the surround is masked with an orthogonal grating. In $A$, the normalized responses from two cells are plotted. Both of these cells were tested with a limited portion of the surround stimulated, based on detailed mapping (see icon 1). These cells display a clear disinhibition effect that exceeds that of the population median with full-field stimulation (open triangles). These results show that it is not necessary to stimulate the entire surround to

disinhibition. The left eye (LE; filled symbols) is dominant and is strongly suppressed by the optimal surround grating. Minor recovery is observed for this eye. For the nondominant right eye ( $R E$; open symbols), the cell fully recovers an optimal response with a high-contrast orthogonal surround grating. The arrowheads on the $x$-axis denote the contrast of the optimal grating. $S A$, Spontaneous activity. 
Figure 3. A summary is shown of disinhibition with full-field optimal gratings and annular, orthogonal gratings (29 data sets). In $A$, the responses from each cell are normalized to the response to the large optimal grating [center plus surround $(C t r+$ Surr $)]$, so 1 on the plot is the baseline response when an optimal grating that covers both the center and surround is presented. The open triangles reflect the median value at each contrast level (slightly offset to the right for visibility). The median gives a more conservative estimate of the effect than the mean, which can be skewed by the cells with large changes in response. The one curve that rises dramatically beginning at $4 \%$ contrast reaches a peak of 29.2 times the suppressed response. This enormous increase in response is attributable partly to an almost complete suppression by the surround. The response of the cell is given in Figure $2 B$. For comparison, the cell in Figure $2 A$ responds 2.1 times greater in the plaid condition compared with the optimal center plus surround condition. The mean and median change in response at $80 \%$ contrast of the orthogonal surround grating is 2.99 and 1.89 , respectively. In $B$, histograms are given of the numbers of cells for which response increased (open bars) or decreased (shaded bars) when an orthogonal grating of different contrasts is superimposed on the optimal grating in the area outside the CRF. In $C$, a suppression index is plotted against a disinhibition index. The suppression index is defined as follows: $\left(R_{\mathrm{C}+\mathrm{S}} /\right.$ $\left.R_{\text {opt }}\right) \times 100 \%$, where $R_{\mathrm{C}+\mathrm{S}}$ is the response to the full-field stimulus covering the center and surround, and $R_{\mathrm{opt}}$ is the response of the cell to the optimal stimulus presented to the center alone. The disinhibition index is defined as follows: $\left(R_{\mathrm{C}+\text { plaid }} / R_{\text {opt }}\right) \times 100 \%$, where $R_{\mathrm{C}+\text { plaid }}$ is the response to the optimal stimulus presented in the center and the plaid stimulus (at $80 \%$ ) presented in the surround. $R_{\text {opt }}$ is as defined above. With this index, a value of 0 indicates no response from the cell, 100 indicates that the response with the plaid is equal to $R_{\mathrm{opt}}$, and values above 100 indicate responses that are stronger than $R_{\text {opt }}$.
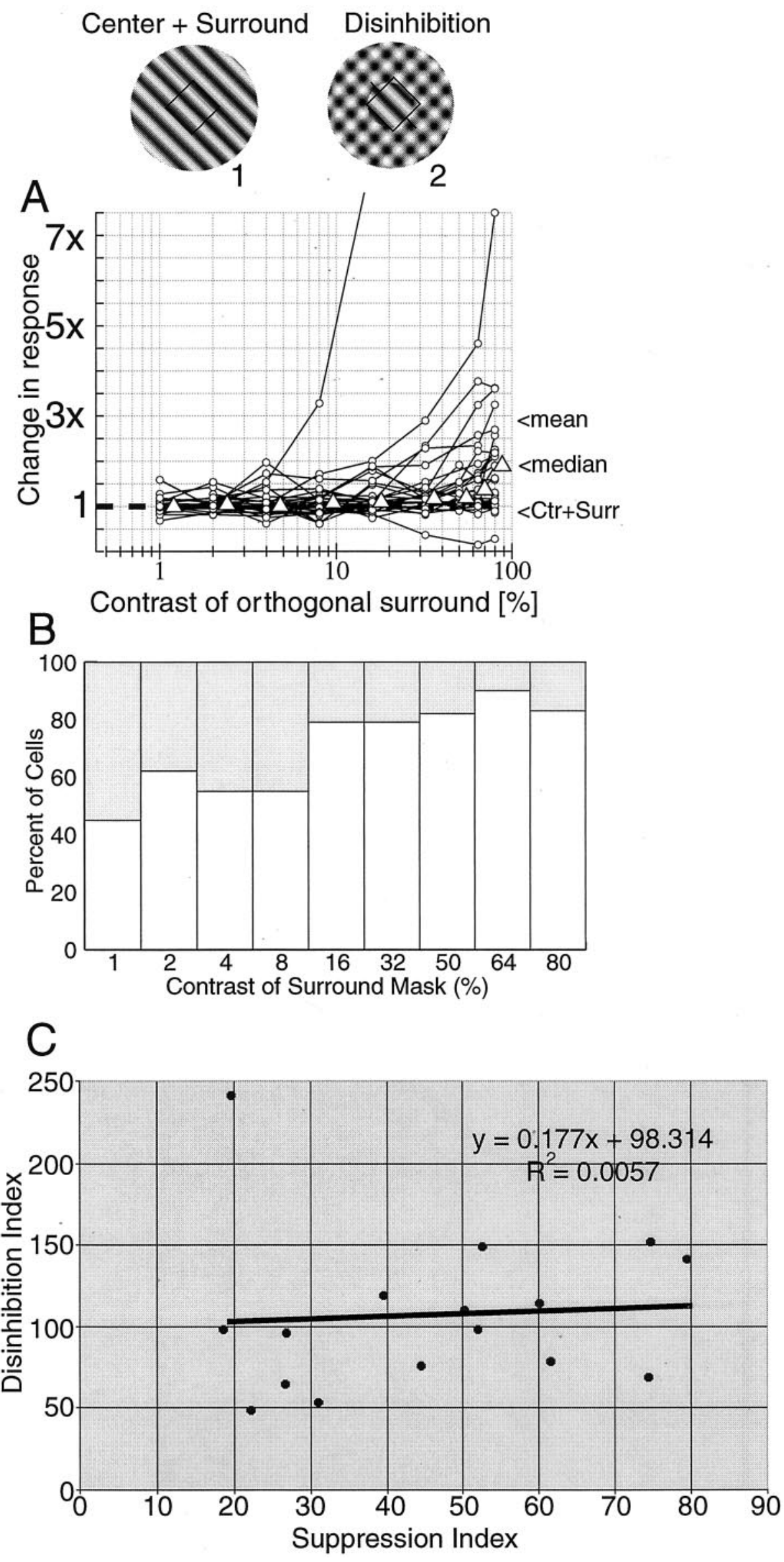


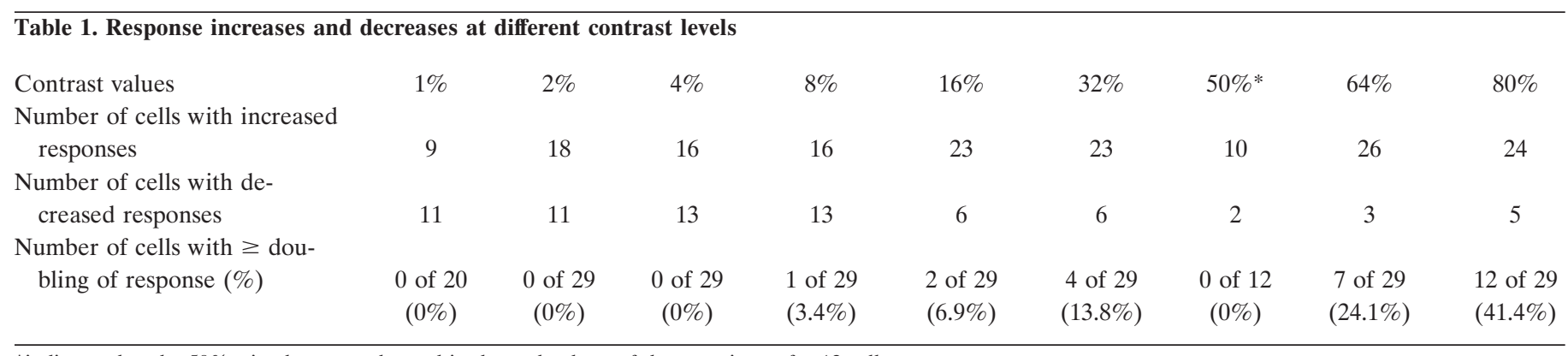

*indicates that the $50 \%$ stimulus was only used in the early phase of the experiment for 12 cells.

get a pronounced effect. Furthermore, there does not need to be a figure-ground component in the image. The stimulus shown here does not convey a pop-out or figure-ground impression.

Another stimulus configuration was tested for three cells for which surround mapping revealed clear spatial asymmetries. We stimulated the entire center and surround with an optimal grating while placing an orthogonal grating over just the inhibitory portion of the surround (Fig. 5, icon 2). With this configuration, the small plaid region forms a locally prominent figure against the background of the large optimal grating. If response is modulated based on the context of the stimulus, the response of the cell should be decreased because the CRF lies in a portion of the image that corresponds to the background. Therefore, as the contrast (and perceptual saliency) of the orthogonal grating increases, the figure-ground model predicts no change in response or even an additional decrease. As the results in Figure $5 B$ demonstrate, this did not occur. On the contrary, disinhibition was observed for all three cells. For comparison, population data from full-field masking conditions are included (open triangles). These data suggest a simple local interaction process without a requirement for context-based images or higher-order feedback. The disinhibition model provides a parsimonious account of the data.

\section{Facilitation or disinhibition?}

The individual cells we studied exhibit varying degrees of disinhibition. Responses do not increase significantly beyond the optimal level for any of the examples shown. To see whether this trend is consistent across the population, we re-normalized the data to the responses obtained with the optimal gratings restricted to the CRF as shown in Figure 6A. Optimal responses were recorded in separate trials immediately after the main test trials, and some cells were lost before the complete set of control measurements were collected, so results for 13 cells are shown. In this plot, 1 represents the response to the optimal stimulus presented to the center alone. Therefore, at the lowest contrasts of the orthogonal grating, one sees the distribution of surround suppression across the population. For low contrasts, there is no change in the suppression. At the higher contrasts, the responses increase, as illustrated in previous figures. As shown in the population mean and median (Fig. $6 B$ ), there is a slight increase above 1 for the highest contrast level.

These results indicate that, although many cells are fully disinhibited, they do not generally respond much stronger than the level obtained with optimal center stimulation alone. The data shown in Figure $6 B$ provide a clear demonstration of the disinhibition effect over the population, with only minor facilitation.

\section{DISCUSSION}

The main finding of this study is that surround suppression may be partially or completely reversed by appropriate stimuli. We found in previous work that nearly all influence from outside the CRF is inhibitory in nature (Walker et al., 1999, 2000). In the current study, we determined that this inhibition may be offset by a stimulus that, by itself, has no effect on the response of the cell. A grating patch that is orthogonal to the preferred orientation of the cell, and is positioned outside the CRF, does not influence the response of the cell. However, when placed over a grating patch of the preferred orientation to form an orthogonal grid, the combination reduces the inhibitory effect, partially or completely. This process of disinhibition may be accounted for by local neural connections, as described below.

\section{Figure-ground segregation}

Considerable interest has been raised by a possible perceptual application of CRF and surround organization. Specifically, it has been proposed that the segregation of a figure from a background is assisted by the suppressive region surrounding the CRF (Nothdurft, 1991; Lamme, 1995; Kastner et al., 1997). This is an appealing idea, but it is also difficult to interpret. The standard figure-ground pattern consists of a central patch of a given pattern that is surrounded by a different pattern, as in the case of the icon of Figure $1 A_{5}$. However, a looser definition could include adjoining patterns that are different, such as those illustrated by the icons in Figure $1 B_{5}$. Another potential difficulty is that figureground processing could occur in one or more of several cortical areas. Therefore, ruling out figure-ground segregation in V1, as reported recently (Rossi et al., 2001), does not eliminate the possibility that this function occurs in other cortical areas. Obviously, because it is a perceptual reality, figure-ground segregation must occur in one or more cortical areas.

The interactions we report in the current study may be accounted for by local processes that are not necessarily connected to perceptual events. For example, we find the same degree of suppression and disinhibition for surround-CRF interaction for a small patch of gratings as for a complete pattern that covers the entire circumference of the CRF. In the classical pattern, a figure is surrounded entirely by the surround. Unless, as noted above, one includes any adjacent pattern differences as a figure-ground pattern, our results may be accounted for on the basis of local events that are not necessarily connected to a perceptual process.

\section{A neural mechanism for disinhibition}

A neural circuit that could form the basis of a disinhibition process is illustrated in Figure 7. This model assumes a spatiotemporal convolution, followed by a contrast normalization process and a static output nonlinearity, as depicted within the 


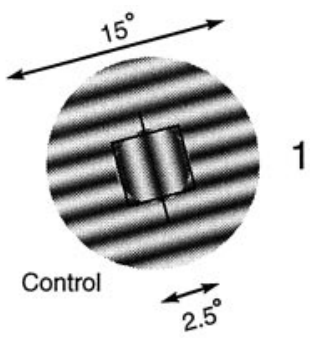

1

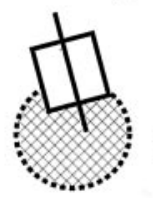

3

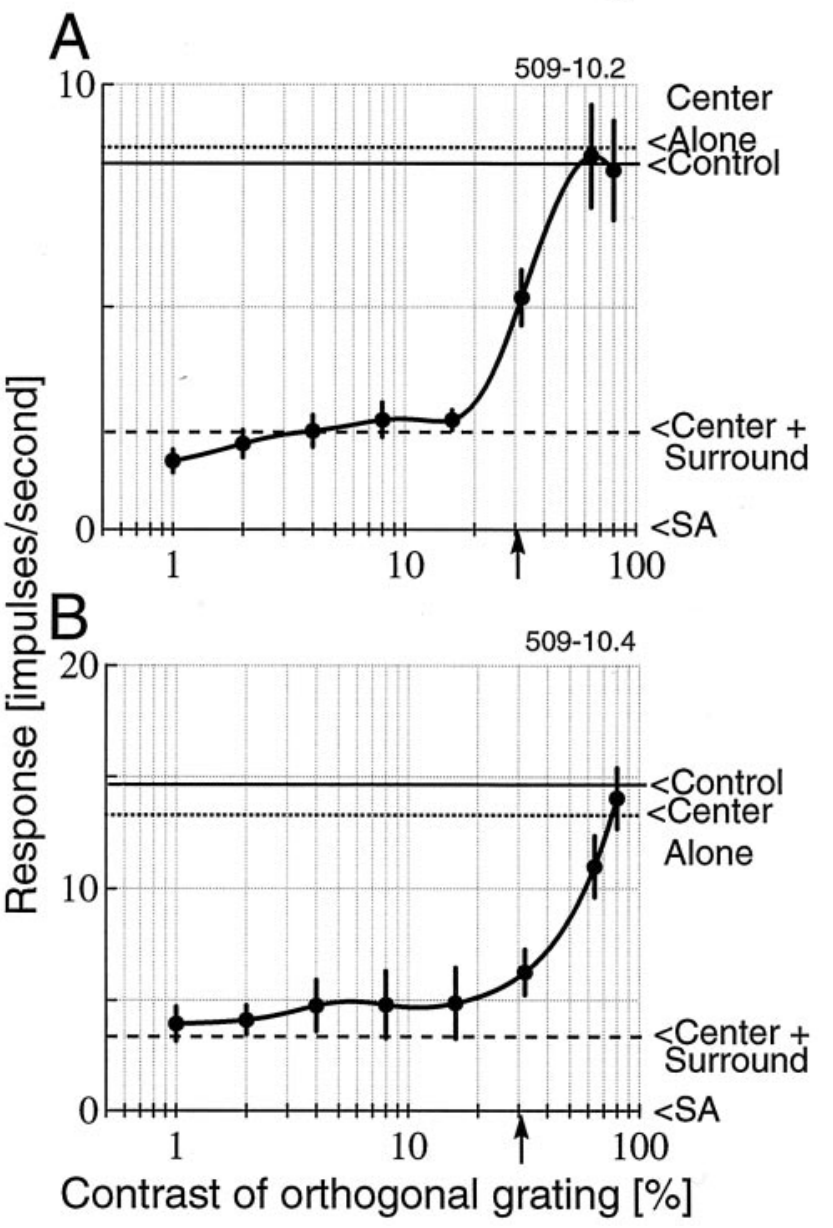

Figure 4. An example is given of disinhibition with two different stimulus configurations for one neuron. $A$, Disinhibition is observed with the full-field optimal grating (CRF plus surround) and annular orthogonal grating surrounding the CRF (icon 2). The control response is the mean response from optimal stimulation limited to the CRF and the orthogonal grating presented to the surround at the various contrast levels used in the test conditions (icon 1). Note that the orthogonal grating, presented alone, has no effect on response of this cell to the optimal center stimulus. $B$, The experiment is repeated, but this time, only the suppressive portion of the surround is used. The surround mapping reveals strong asymmetric suppression predominantly from the bottom end of the RF (icon 3, which shows the CRF and a hatched inhibitory region). The actual stimulus configuration used is shown in icon 4 . The responses show total disinhibition of the suppression. For this run, the control condition consists of optimal stimulation of the center and orthogonal grating stimulation over the bottom of the surround. The contrast of the optimal grating is $30 \%$ in both cases and is indicated by arrows on the $x$-axis.
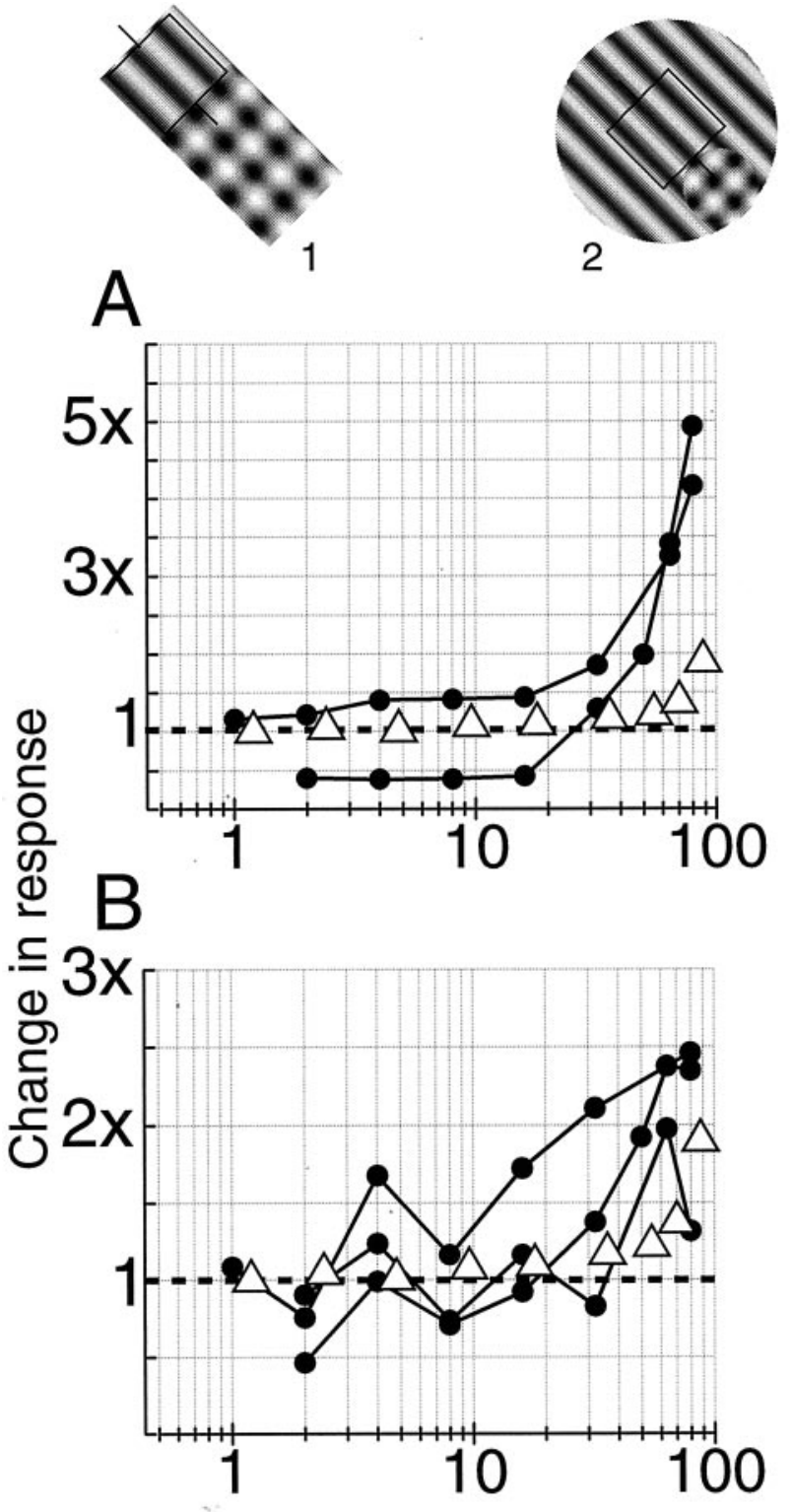

\section{Contrast of orthogonal surround [\%]}

Figure 5. A summary is shown of cells tested with the orthogonal grating limited to small portions of the surround. In $A$, normalized responses from two cells are illustrated, along with the population median data (open triangles) for the full-field condition. These cells were tested with rectangular grating patches that extended only into the suppressive portion of the surround (icon 1). Both cells exhibit marked disinhibition that exceeds the median population change. $B$, Normalized responses are shown from three cells tested with the configuration shown in icon 2. In these cases, the optimal grating covers the entire center and surround, whereas the orthogonal grating covers only the suppressive portion of the surround. The open triangles are the population median values.

rectangle of Figure 7 (Heeger, 1992; Carandini et al., 1997). We assume that this process is characteristic of all striate cortex RFs in the entire visual field. We also assume that, for certain cells, there is a subset of neurons with similar RF properties that serves as the basis of surround suppression for a given cell. The example in Figure 7 arbitrarily uses four cells in the surround pool. They are all spatially overlapping but displaced from the central RF, and all four cells prefer orientations near vertical, as does the cell 


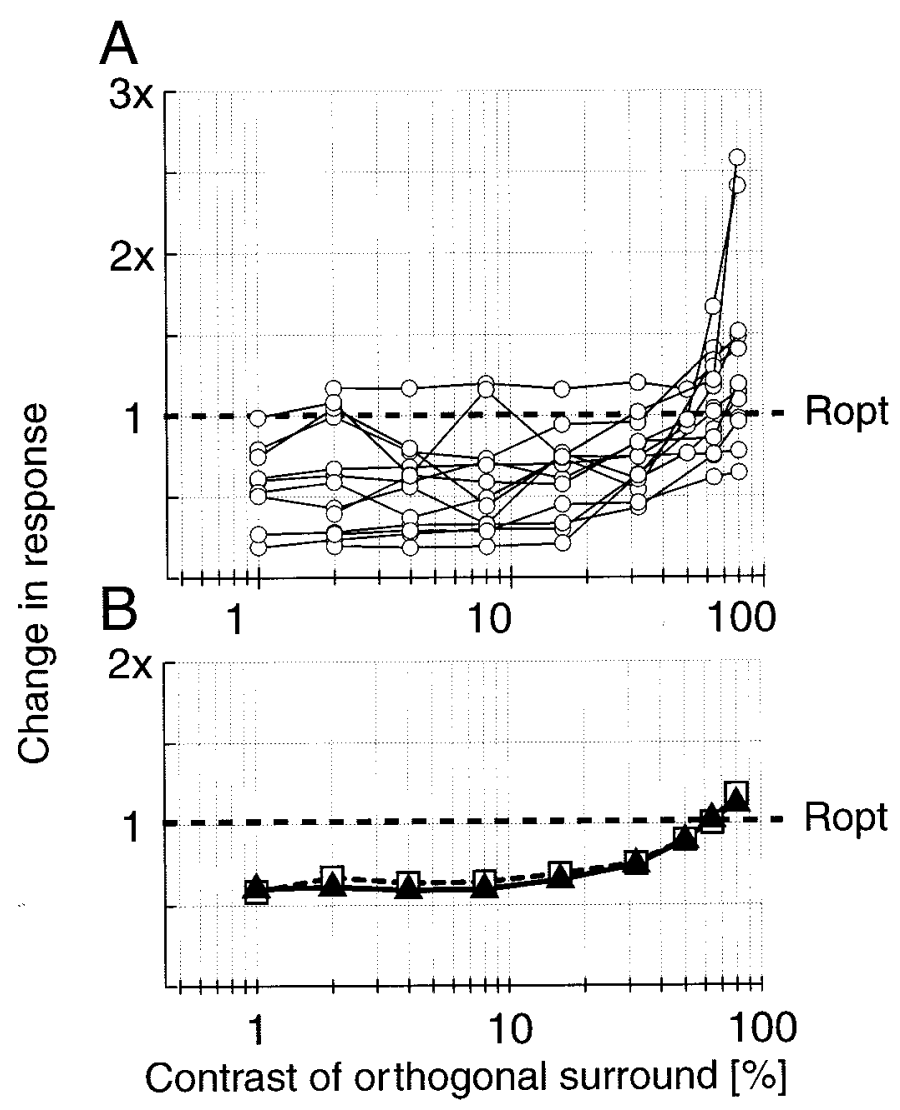

Figure 6. A summary is given of normalized responses from cells for which appropriate control data are available. The responses are all normalized to the response of the optimal grating restricted to the CRF. $A$, The responses to $1 \%$ orthogonal grating contrast indicate the distribution of surround suppression for the population tested. The mean and median of this distribution are essentially unchanged until $\sim 16 \%$ contrast, and then they increase monotonically. At the highest contrast (80\%), there is an overall tendency for slight facilitation above the responses to the optimal center stimulus. $B$, The population mean (open squares) and median (filled triangles) values are shown here.

in the center (DeAngelis et al., 1992). The cells in the surround could inhibit the center cell directly through monosynaptic inhibition or they could act through interneurons.

We assume a contrast normalization process for the CRF. Contrast normalization exhibits two prominent properties. First, it is divisive. Second, the signal that serves as the denominator in the division is proportional to the total contrast energy of the visual stimulus within a specified region of space (Heeger, 1992; Carandini et al., 1999). It has been suggested that the surround may act as an extension of the contrast normalization mechanism in the CRF (Cavanaugh et al., 1998). Consistent with this idea is the observation that, with increasing contrast of iso-oriented surround gratings, the contrast response function of the center shifts to the right on log coordinates (Cavanaugh et al., 1998). This is the expected result of a divisive operation or contrast normalization (Heeger, 1992; Cavanaugh et al., 1998; Carandini et al., 1999). In addition, iso-oriented surround suppression increases proportionately with contrast (Cavanaugh et al., 1998). This is also consistent with a normalization process. However, other aspects of surround suppression are not consistent with contrast normalization. For example, surround suppression is generally tuned to orientations and spatial frequencies that match the preferred values of the CRF (Nelson and Frost, 1978; DeAn-

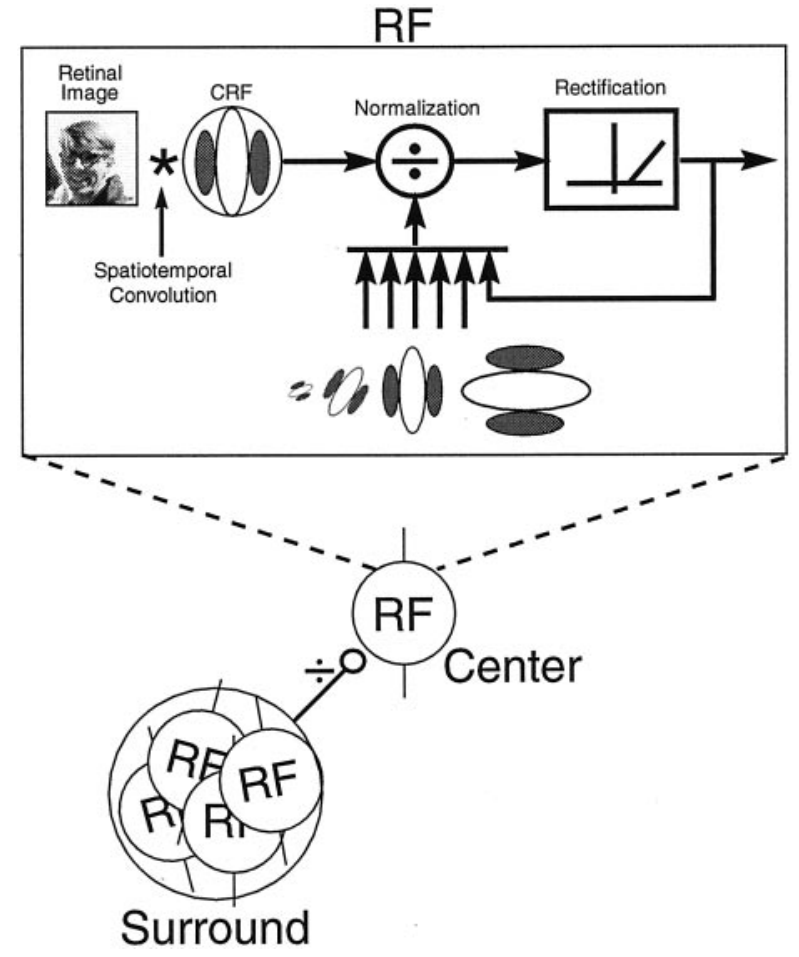

Figure 7. A schematic illustration is presented of a model that provides the underlying neural circuitry of local contrast normalization and surround suppression. Each RF is assumed to exhibit normalization, with broadband rectification underlying its response properties. This includes the cell designated as the Center, as well as those cells contributing to the Surround. The pool of cells contributing to surround suppression are spatially displaced from the center RF but are tightly clustered and have similar orientation preferences to that of the center cell.

gelis et al., 1994; Li and Li, 1994), whereas contrast normalization in the CRF is presumed to pool over the entire range of orientations (Heeger, 1992). In addition, our current results suggest that the cells comprising the surround of the CRF are themselves subject to local broadband contrast normalization. When an orthogonal grating is added to the surround, the total contrast energy in the stimulus increases. However, contrary to the prediction of a general contrast normalization mechanism, our results show that most cells respond more vigorously to this added contrast energy. Considered together, these findings suggest that the center plus surround should not be considered as a pool for a combined contrast normalization.

\section{The nature of influence from the surround}

A fundamental question that must be addressed concerns the nature of the influence from the region beyond the CRF. Several reports suggest that surround stimulation can increase the response of the cell (Maffei and Fiorentini, 1976; Sillito et al., 1995; Levitt and Lund, 1997; Polat et al., 1998). This means that activation of the surround excites a cell to respond above the level obtained with optimal stimulation of the center. This has been reported to occur even for stimulation in the CRF with a grating that is orthogonal to the preferred orientation (Sillito et al., 1995).

In general, we observed very limited facilitation from the surround (Walker et al., 1999, 2000). We find that the surround modulates the excitation generated by stimulation of the CRF. In extensive tests, we found that apparent facilitation by stimulation of the surround is nearly always caused by faulty centering of the 
grating such that part of the stimulus is delivered to the CRF (Walker et al., 1999, 2000).

It is important to distinguish the results of disinhibition that we report here from those of facilitation. In the case of disinhibition, increased response strength results from a withdrawal of inhibition. Another factor that could play a role in this process is tonic inhibition from cells whose RFs are in the surround. If a grating that is orthogonal to the optimal orientation silences the surround cells, then the source of tonic inhibition is removed, and this would increase the response to CRF stimulation.

An advantage of the plaid surround used in our current study is that the contrast energy of the optimal surround grating remains constant throughout the tests. The addition of the orthogonal grating simply adds contrast energy in another part of the spectrum. There is still sufficient contrast energy in the stimulus to activate surround suppression. The resultant disinhibition shows that responses of cells providing surround suppression are masked by the orthogonal grating.

Finally, it should be mentioned that the choice of a response reference frame can influence the interpretation of a given set of findings. In some figure-ground studies, the response to a fullfield homogeneous texture stimulus is taken as the baseline (Lamme, 1995; Zipser et al., 1996). This means that the baseline response consists of excitation from the CRF and suppression from the surround. The overall result is that the baseline response is lower than that of the CRF alone. If the cell is then tested with texture restricted to the CRF, with or without texture in the surround, this could be interpreted as facilitation. An additional interpretation is that the facilitated response is attributable to a restricted stimulus, i.e., a figure, standing out as perceptually relevant against a background. If an alternative baseline is used consisting of stimulation of the CRF alone, then manipulations of the surround would not influence the response of the cell, except to reduce it.

\section{REFERENCES}

Allman J, Miezin F, McGuinness E (1985) Stimulus specific responses from beyond the classical receptive field: neurophysiological mechanisms for local-global comparisons in visual neurons. Annu Rev Neurosci 8:407-430.

Anzai A, Ohzawa I, Freeman RD (1997) Neural mechanisms underlying binocular fusion and stereopsis: position vs. phase. Proc Natl Acad Sci USA 94:5438-5443.

Bonds AB (1989) Role of inhibition in the specification of orientation selectivity of cells in the cat striate cortex. Vis Neurosci 2:41-55.

Butler TW, Westheimer G (1978) Interference with stereoscopic acuity: spatial, temporal, and disparity tuning. Vision Res 18:1387-1392.

Carandini M, Heeger DJ, Movshon JA (1997) Linearity and normalization in simple cells of the macaque primary visual cortex. J Neurosci $17: 8621-8644$.

Carandini M, Heeger DJ, Movshon JA (1999) Linearity and gain control in V1 simple cells. In: Models of cortical circuits. Cerebral cortex, Vol 13 (Ulinski PS, Jones EG, Peters A, eds), pp 3-31. New York: Plenum.
Cavanaugh JR, Bair W, Movshon JA (1998) Signals setting contrast gain arise from iso-oriented domains aligned with the receptive field axis of macaque striate cortex neurons. Soc Neurosci Abstr 24:1875.

DeAngelis GC, Robson JG, Ohzawa I, Freeman RD (1992) Organization of suppression in receptive fields of neurons in cat visual cortex. J Neurophysiol 68:144-163.

DeAngelis GC, Ohzawa I, Freeman RD (1993) Spatiotemporal organization of simple-cell receptive fields in the cat's striate cortex. II. Linearity of temporal and spatial summation $J$ Neurophysiol 69:1118-1135.

DeAngelis GC, Freeman RD, Ohzawa I (1994) Length and width tuning of neurons in the cat's primary visual cortex. J Neurophysiol 71:347-374.

Heeger DJ (1992) Normalization of cell responses in cat striate cortex. Vis Neurosci 9:181-197.

Hubel DH, Wiesel TN (1962) Receptive fields, binocular interaction and functional architecture in the cat's visual cortex. J Physiol (Lond) 160:106-154.

Hupe JM, James AC, Payne BR, Lomber SG, Girard P, Bullier J (1998) Cortical feedback improves discrimination between figure and background by V1, V2 and V3 neurons. Nature 394:784-787.

Kastner S, Nothdurft HC, Pigarev IN (1997) Neuronal correlates of pop-out in cat striate cortex. Vision Res 37:371-376.

Knierim JJ, van Essen DC (1992) Neuronal responses to static texture patterns in area V1 of the alert macaque monkey. J Neurophysiol 67:961-980

Lamme VA (1995) The neurophysiology of figure-ground segregation in primary visual cortex. J Neurosci 15:1605-1615.

Levitt JB, Lund JS (1997) Contrast dependence of contextual effects in primate visual cortex. Nature 387:73-76.

Li CY, Li W (1994) Extensive integration field beyond the classical receptive field of cat's striate cortical neurons-classification and tuning properties. Vision Res 34:2337-2355.

Maffei L, Fiorentini A (1976) The unresponsive regions of visual cortical receptive fields. Vision Res 16:1131-1139.

Morrone MC, Burr DC, Maffei L (1982) Functional implications of cross-orientation inhibition of cortical visual cells. I. Neurophysiological evidence. Proc R Soc Lond B Biol Sci 216:335-354.

Nelson JI, Frost BJ (1978) Orientation-selective inhibition from beyond the classic visual receptive field. Brain Res 139:359-365.

Nothdurft HC (1991) Texture segmentation and pop-out from orientation contrast. Vision Res 31:1073-1078.

Pelli DG, Zhang L (1991) Accurate control of contrast on microcomputer displays. Vision Res 31:1337-1350.

Polat U, Mizobe K, Pettet MW, Kasamatsu T, Norcia AM (1998) Collinear stimuli regulate visual responses depending on cell's contrast threshold. Nature 391:580-584.

Rossi AF, Desimone R, Ungerleider LG (2001) Contextual modulation in primary visual cortex of macaques. J Neurosci 21:1698-1709.

Sillito AM, Grieve KL, Jones HE, Cudeiro J, Davis J (1995) Visual cortical mechanisms detecting focal orientation discontinuities. Nature 378:492-496.

Skottun BC, De Valois RL, Grosof DH, Movshon JA, Albrecht DG, Bonds AB (1991) Classifying simple and complex cells on the basis of response modulation. Vision Res 31:1079-1086.

Sutter EE, Tran D (1992) The field topography of ERG components in man. I. The photopic luminance response. Vision Res 32:433-446.

Walker GA, Ohzawa I, Freeman RD (1998) Binocular cross-orientation suppression in the cat's striate cortex. J Neurophysiol 79:227-239.

Walker GA, Ohzawa I, Freeman RD (1999) Asymmetric suppression outside the classical receptive field of the visual cortex. J Neurosci 19:10536-10553.

Walker GA, Ohzawa I, Freeman RD (2000) Suppression outside the classical cortical receptive field. Vis Neurosci 17:369-379.

Zipser K, Lamme VA, Schiller PH (1996) Contextual modulation in primary visual cortex. J Neurosci 16:7376-7389. 